\title{
Analytical Calculation of Magnet Systems: Magnetic Field Created by Charged Triangles and Polyhedra
}

\author{
Christophe Rubeck ${ }^{1}$, Jean-Paul Yonnet ${ }^{1}$, Hicham Allag ${ }^{1,2}$, Benoît Delinchant ${ }^{1}$, and Olivier Chadebec ${ }^{1}$ \\ ${ }^{1}$ Laboratoire de Génie Electrique de Grenoble, UMR 5269 CNRS/INPG/UJF, Institut Polytechnique de Grenoble, Saint Martin \\ d'Hères, 38402 France \\ ${ }^{2}$ University of Jijel, Algeria
}

\begin{abstract}
An analytical method for the calculation of the magnetostatic scalar potential and the magnetic field created by a polyhedron-shaped permanent magnet is presented in this paper. The magnet is supposed to be uniformly magnetized. The magnetization is equivalent to distributions of magnetic charges: it is the coulombian approach. The analytical calculation is made by a surface integration on all the polygons that composes the polyhedron. For each polygonal surface, we have shown that it can be decomposed in a series of right triangles. An analytical solution in the particular case of the right triangle has been developed. By this way, the magnetostatic potential and the magnetic field of any polyhedral-shaped magnet can be analytically calculated.

The analytical results have been verified: the field calculation created by a prismatic magnet is developed.
\end{abstract}

Index Terms-Analytical calculation, magnetic charges, magnetic field, permanent magnet.

\section{INTRODUCTION}

$\mathbf{T}$ HERE are a lot of analytical models for the calculation of field and strength of permanent magnets in the literature for a wide variety of shaped magnets (parallelepipeds [1], [2]; cylinders [3], [4]; rings [5], [6]; and tiles [7], [8]). But some magnets own a more complicated shape. An analytical method for calculating the scalar potential and the magnetic field created by a magnet of any polyhedral shape [see Fig. 1(A)] is presented in this paper.

In 2010, J. L. G. Janssen has presented a method for the calculation of the field of prismatic magnets [9]. The work is based on a similar decomposition, but the field expressions are slightly different because the origin has been taken in the middle of the triangle hypotenuse, when the origin is at a triangle corner in our work [10].

\section{Calculation Principle}

The analytical calculation of the scalar potential and the magnetic field created by a permanent magnet is done by a coulombian approach. It means that the magnetization in the volume of the material is represented by a distribution of magnetic charges on the surface of this volume (Fig. 1(B)).

The magnet is supposed to be perfect. It means that the magnetization is constant and uniform, with a permeability of $\mu_{0}$.

The first step of the calculation is to reduce the polyhedron into polygons. The magnetic charges are caused by the discontinuity of the normal component of the magnetization on the surface. Therefore it gives $\sigma_{m}=\boldsymbol{J} \cdot \boldsymbol{n}$, where $\sigma_{m}$ is the magnetostatic surface charge density, $\boldsymbol{J}$ is the magnet polarization in Tesla, and $\boldsymbol{n}$ is the normal vector to the surface of the magnet.
A

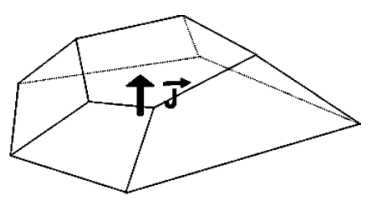

B

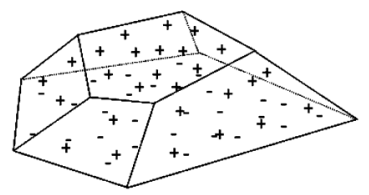

Fig. 1. Example of polyhedron-shaped magnet. (A) Constant and uniform magnetization, $J$ is the polarization in Tesla.. (B) Equivalent coulombian representation (surface distribution of magnetic charges).

The magnetic scalar potential $\Phi(r)$ is given by the following integral [11]

$$
\Phi(r)=\frac{\sigma_{m}}{4 \pi \mu_{0}} \iint_{S} \frac{1}{r} d S .
$$

The geometrical parameter $r$ is the distance between the considered point where the potential is calculated and the magnetic charges.

The magnetic field $H(r)$ is written by [11]

$$
\vec{H}(\vec{r})=-\vec{\nabla} \Phi(r)=\frac{\sigma_{m}}{4 \pi \mu_{0}} \iint_{S} \frac{\vec{r}}{r^{3}} d S .
$$

The analytical calculation of the magnetostatic scalar potential or the magnetic field created by a uniformly charged surface of any polygonal shape is not trivial. Indeed, there is no generic solution of the integral (1) and (2) on any polygons. However, an analytical solution can be obtained in a particular case: a right triangle. The configuration of the right triangle is presented in Fig. 2. The integration point is located on a line crossing perpendicularly the triangle plane in one of the nonright-angled vertices of the right triangle. From the solution for the right triangle, the calculation method is based on the reduction of any polygon in a series of triangles [12]. 


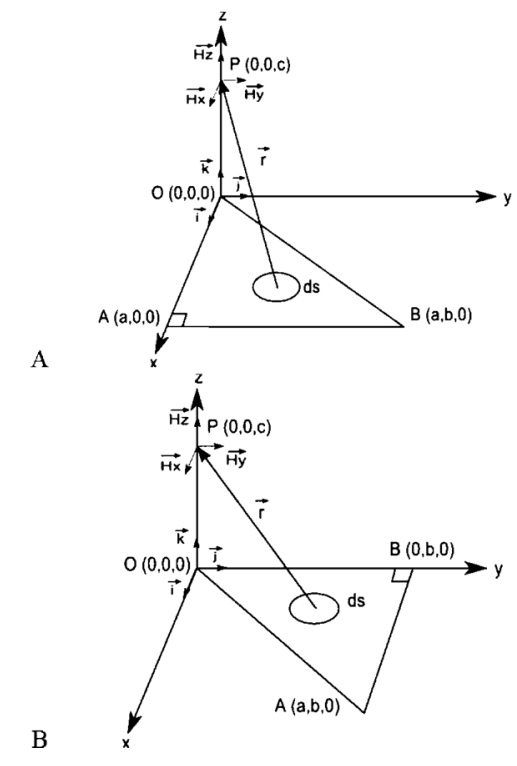

Fig. 2. Definition of the two types of right triangles: (A) right angle is located on $O x$ and (B) right angle is on $O y$.

In the paper, the analytical expressions of the potential and magnetic field components on right triangles are given. Then the decomposition of a polygonal charged surface in a series of right triangle reductions is presented. The method is illustrated on a simple prismatic magnet and the results are compared with a numerical simulation by finite elements.

\section{Calculation of the Potential and the Magnetic} Field CReated By a Uniformly Charged Right TRIangle

Let the right triangle shown in Fig. 2(A) be uniformly charged. The magnetic potential and the field components are given by

$$
\begin{aligned}
\Phi(r) & =\frac{\sigma_{m}}{4 \pi \mu_{0}} \iint_{S} \frac{1}{r} d S \\
& =\frac{\sigma_{m}}{4 \pi \mu_{0}} \int_{x=0}^{x=a} \int_{y=0}^{y=b x / a} \frac{1}{\sqrt{x^{2}+y^{2}+c^{2}}} d x d y \\
H_{x}(r) & =\frac{\sigma_{m}}{4 \pi \mu_{0}} \iint_{S} \frac{\vec{r} \cdot \vec{i}}{r^{3}} d S \\
& =\frac{\sigma_{m}}{4 \pi \mu_{0}} \int_{x=0}^{x=a} \int_{y=0}^{y=b x / a} \frac{-x}{\left[x^{2}+y^{2}+c^{2}\right]^{3 / 2}} d x d y \\
H_{y}(r) & =\frac{\sigma_{m}}{4 \pi \mu_{0}} \iint_{S} \frac{\vec{r} \cdot \vec{j}}{r^{3}} d S \\
& =\frac{\sigma_{m}}{4 \pi \mu_{0}} \int_{x=0}^{x=a} \int_{y=0}^{y=b x / a} \frac{-y}{\left[x^{2}+y^{2}+c^{2}\right]^{3 / 2}} d x d y \\
H_{z}(r) & =\frac{\sigma_{m}}{4 \pi \mu_{0}} \iint_{S} \frac{\vec{r} \cdot \vec{k}}{r^{3}} d S \\
& =\frac{\sigma_{m}}{4 \pi \mu_{0}} \int_{x=0}^{x=a} \int_{y=0}^{y=b x / a} \frac{c}{\left[x^{2}+y^{2}+c^{2}\right]^{3 / 2}} d x d y
\end{aligned}
$$

where $\vec{r}=-x \cdot \vec{i}-y \cdot \vec{j}+c \cdot \vec{k}$.
A direct calculation of (3), (4), (5), and (6) gives the following analytical expressions:

$$
\begin{aligned}
\Phi(r)= & \frac{\sigma_{m}}{4 \pi \mu_{0}}\left\{\frac{a}{2} \ln \left(\frac{D_{a b c}+b}{D_{a b c}-b}\right)\right. \\
& \left.-|c| \arctan \left(\frac{a b}{D_{a c}^{2}+|c| D_{a b c}}\right)\right\} \\
H_{x}(r)= & \frac{\sigma_{m}}{4 \pi \mu_{0}}\left\{\frac{-b}{2 D_{a b}} \ln \left(\frac{D_{a b c}+D_{a b}}{D_{a b c}-D_{a b}}\right)\right. \\
& \left.+\frac{1}{2} \ln \left(\frac{D_{a b c}+b}{D_{a b c}-b}\right)\right\} \\
H_{y}(r)= & \frac{\sigma_{m}}{4 \pi \mu_{0}}\left\{\frac{a}{2 D_{a b}} \ln \left(\frac{D_{a b c}+D_{a b}}{D_{a b c}-D_{a b}}\right)\right. \\
& \left.-\frac{1}{2} \ln \left(\frac{D_{a c}+a}{D_{a c}-a}\right)\right\} \\
H_{z}(r)= & \frac{\sigma_{m}}{4 \pi \mu_{0}}\left\{\arctan \left(\frac{a D_{a b c}}{b c}\right)\right. \\
& \left.-\frac{|c|}{c} \arctan \left(\frac{a}{b}\right)\right\}
\end{aligned}
$$

with $D_{a b c}=\sqrt{a^{2}+b^{2}+c^{2}}, D_{a b}=\sqrt{a^{2}+b^{2}}$, and $D_{a c}=$ $\sqrt{a^{2}+c^{2}}$.

The expressions associated with the right triangle shown in Fig. 2(B) are analogical to (7)-(10). They can be found in considering a set of rotations of the right triangle first around $\mathrm{Oz}$ axis then around $O y$ axis.

Note: The normal component of the magnetic field $H_{z}$ is discontinuous in crossing the surface $S$. It is reasonable to take $H_{z}=0$ for $c=0$ [11]. It must also be pointed out that the field components $H_{x}$ and $H_{y}$ are undefined for $c=0$ (the plane of the polygon). The divergent terms cancel analytically when all the right triangles of a polygon are combined together. It was analytically verified on the case of the rectangle and numerically for other polygonal shapes (the divergent terms are simply not calculated).

It is now possible to calculate the magnetic scalar potential and field created by a magnet of any polygonal section by reducing it into a sum of right triangles of kind $\mathrm{A}$ and $\mathrm{B}$.

\section{Reduction of an Integral on a Polygon Into a Sum OF INTEGRALS ON RIGHT TRIANGLES}

Let be an n edge polygon, $P$ is the integration point and $O$ is its orthogonal projection in the plane of the polygon. The triangles associated with the edges of the polygon and point $O$ can be generated to the maximum number of $n$ [13]. Indeed there will be $n-1$ triangles if $O$ is located on an edge of the polygon and $n-2$ if $O$ is located on a top. Each of these triangles is then reduced into a maximum of two right triangles [14]. Therefore the reduction of an $n$ side polygon generates a maximum of $2 n$ right triangles.

The steps of the reduction are shown for the $A B C$ triangle (Fig. 3(A), (B), (C), and (D)). The projection of $O$ on an edge generates two right triangles. Each triangle wears a charge density.

The integral on $A B C$ is the integral over $O B C$ added to the integral over $O C A$ subtracted to the integral over $O A B$ [see Fig. 3(D)]. For example the calculation of the integral over $O B C$ [see Fig. 3(B)] needs the integral associated with the right 


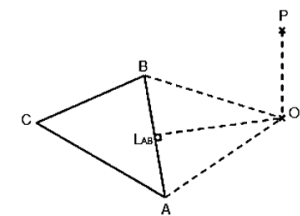

A

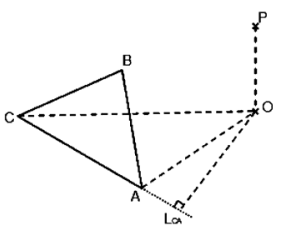

C
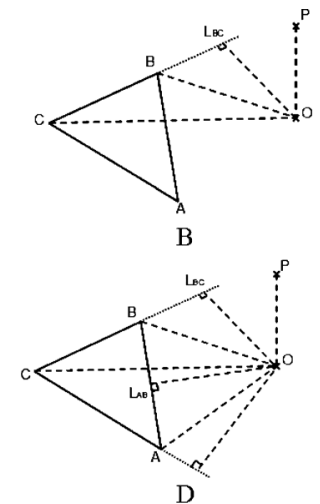

Fig. 3. Reduction of the integration of $A B C$ from the point $P$ to a sum of integrals on right triangles.
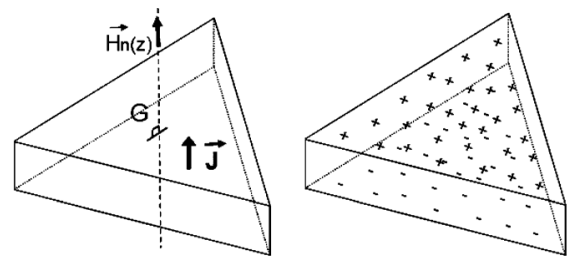

A
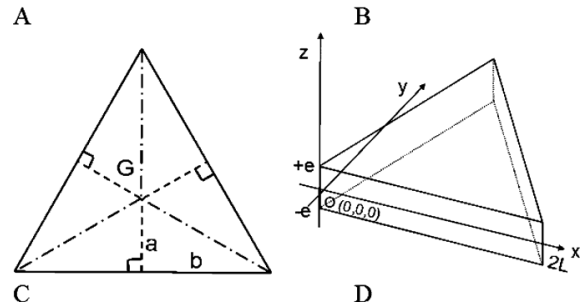

Fig. 4. Steps of the calculation of the normal field above the barycentre of an equilateral triangular base prismal magnet. (A) Equilateral triangular base prismal magnet. (B) Equivalent Coulombian representation. (C) Reduction to right triangles from the barycenter. (D) Sizes of the prism.

triangle $C L_{B C} O$ that is counted positively and the one associated with $B L_{B C} O$ that is counted negatively. We can deduce a general rule: the solution of the integral associated with one right triangle is counted positively if the right triangle covers (or covers partially) the triangle $A B C$. Otherwise it is counted negatively. The sum of all the integrals associated with all right triangles, taking into account the signs, is equivalent to the integral over the triangle $A B C$.

\section{Application 1: Calculation of the Magnetic Field CREATEd By A PRismatic MAgnet}

Above the center of an equilateral charged triangle, it is easy to calculate the vertical component of the magnetic field.

Let us consider a prism magnet, magnetized in its thickness direction. The prism section is an equilateral triangular based prism shown in Fig. 4(A), let us calculate the normal field along a path perpendicular to the prism and crossing its barycentre $G$.

1) Calculation of Charge Densities on the Prism Faces: The calculation of charge densities is immediate [see Fig. 4(B)], we obtain $\sigma_{m}= \pm J$ (norm of vector $\boldsymbol{J}$ ). The charge density is positive at the top (North pole+) and negative below (South pole-). The other sides do not wear any charge.

2) Reduction of Charged Faces Into Right Triangles: The reduction into right triangles is trivial; in fact we get on each face

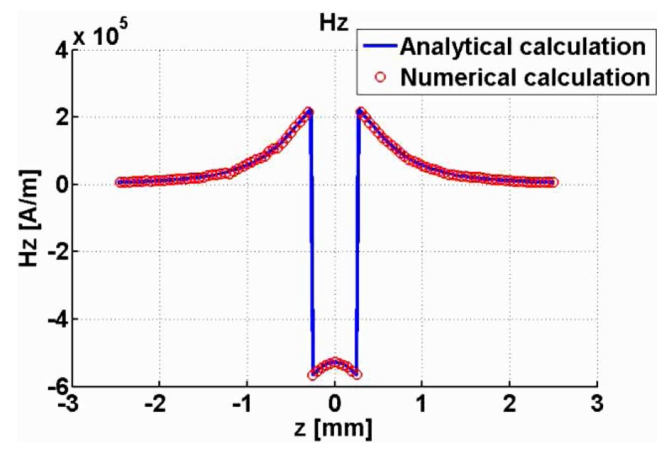

Fig. 5. Normal field $H_{z}$ of the prismatic magnet along a vertical line through the barycentre.

six identical right triangles [see Fig. 4(C)]. In the particular case of the calculation of the normal field, it is not even necessary to conduct an analysis to determine of what kind are the right triangles (Fig. 4, corner of $O x$ or $O y$ ), they are equivalent here. For a prism of $2 L$ width and $2 e$ thickness [see Fig. 4(D)] the parameters of the right triangles are

$$
\begin{aligned}
a & =\frac{1}{3} h, \quad \text { with } h=\frac{\sqrt{3}}{2}(2 L), \quad \text { so } a=\frac{L}{\sqrt{3}}, \\
b & =L, \quad c=z \pm e .
\end{aligned}
$$

The parameter $c$ depends on the face (top or bottom) of the prism that is considered.

3) Analytical Expression of the Normal Field: The normal field is the sum of contributions from 12 charged right triangles ( 6 for the top face and 6 more for the below one). In addition, the right triangles of each face are identical. So according to (10) we obtain (12)

$$
\begin{aligned}
& H_{z}(z)=\frac{6 J}{4 \pi \mu_{0}} \\
& \qquad\left\{\begin{array}{l}
\arctan \left(\frac{\sqrt{\frac{4}{3} L^{2}+(z-e)^{2}}}{\sqrt{3}(z-e)}\right)-\arctan \left(\frac{\sqrt{\frac{4}{3} L^{2}+(z+e)^{2}}}{\sqrt{3}(z+e)}\right) \\
+\left(\frac{|z+e|}{z+e}-\frac{|z-e|}{z-e}\right) \arctan \left(\frac{1}{\sqrt{3}}\right)
\end{array}\right\} .
\end{aligned}
$$

4) Numerical Application and Validation: The sizes of the prism are $L=1 \mathrm{~mm}$ for the 3 edges of the equilateral triangle, and $e=0.25 \mathrm{~mm}$ for the thickness.

The magnet polarization $J$ is taken equal to 1 Tesla. Fig. 5 shows the normal field as a function of $z$ on the vertical line through the barycentre. The obtained results are confronted to a classical numerical simulation by finite elements. The two curves perfectly match. The small defects observed in the numerical calculation are due to the meshing. Note also that the jump at the discontinuity of the field (crossing of the charged surfaces) is $8 \cdot 10^{5} \mathrm{~A} / \mathrm{m}$, which corresponds to the value of $J / \mu_{0}$; it is a classical result.

5) Synthesis: We presented a simple example of field calculation in details. We first calculated the charge distributions on the faces of the prisms. Then we calculated the field $H_{z}$ thanks to a reduction of the charged surfaces into right triangles. The results are then compared to a finite elements modeling. The analytical calculation is very fast and does not require a lot of memory in comparison with a finite element modeling. The 


$$
H z=\frac{\sigma_{m}}{4 \pi \mu_{0}}\left\{\begin{array}{c}
\arctan \left(\frac{\left(x_{0}-m\right)\left(y_{0}-n\right)}{z_{0} \sqrt{\left(x_{0}-m\right)^{2}+\left(y_{0}-n\right)^{2}+z_{0}^{2}}}\right)-\arctan \left(\frac{\left(x_{0}-m\right)\left(y_{0}+n\right)}{z_{0} \sqrt{\left(x_{0}-m\right)^{2}+\left(y_{0}+n\right)^{2}+z_{0}^{2}}}\right) \\
-\arctan \left(\frac{\left(x_{0}+m\right)\left(y_{0}-n\right)}{z_{0} \sqrt{\left(x_{0}+m\right)^{2}+\left(y_{0}-n\right)^{2}+z_{0}^{2}}}\right)+\arctan \left(\frac{\left(x_{0}+m\right)\left(y_{0}+n\right)}{z_{0} \sqrt{\left(x_{0}+m\right)^{2}+\left(y_{0}+n\right)^{2}+z_{0}^{2}}}\right)
\end{array}\right\}
$$

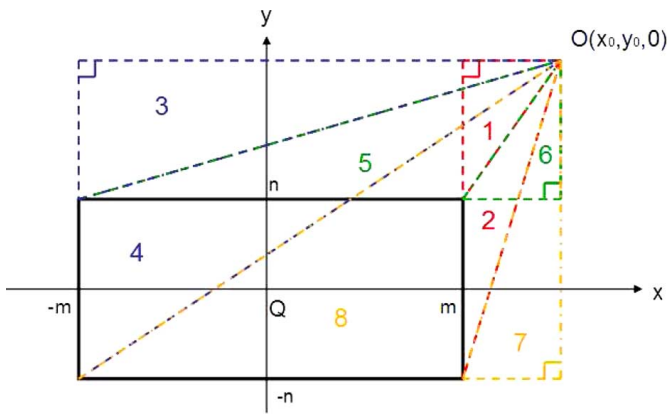

Fig. 6. Reduction of one integral over a rectangle into a sum of integrals over right triangles.

method can be applied on all magnet shapes that can be approximated by a polyhedron.

The calculation of the tangential field $\left(H_{x}\right.$ and $H_{y}$ components) is not presented here because it is a bit more complicated; it requires the projection of each field component from the right triangles coordinate system (Fig. 2) to the global coordinate system. However a computer script that realizes the calculation is easy to perform.

\section{Vi. Application 2: Calculation of the Magnetostatic Field CREATEd By a Right Cuboidal MAGNeT}

A right cuboid is a particular type of polyhedron, with only rectangular faces and right angles. The analytical expressions of the field created by a rectangular surface are a very classical result [1]. The verification of the triangle method has been fully performed on a rectangular surface: the analytical expressions of the magnetic field component calculated by the right triangles method are exactly the same than the analytical expressions obtained by a direct integration.

The first step is the geometrical reduction of the rectangle in right triangles in the plane of the rectangle (Fig. 6). Let $O$ be the orthogonal projection of the integration point in the plane $Q x y$. Each right triangle is labeled according to the parameters of the triangles depending of the kind (corners on $O x$ or $O y$ ).

Only the vertical component is presented here, but the three components can be easily calculated. The $H_{z}$ field component is given by (13), shown at the top of the page.

\section{CONCLUSION}

An analytical calculation method of the scalar potential and the magnetic field created by a polyhedron-shaped permanent magnet has been presented. Thanks to a Coulombian representation of the magnet, the magnetization of the material is consid- ered as a distribution of charges on each face of the polyhedron. Then it is necessary to calculate the integrals of the potential and magnetic field on each of its surfaces. This calculation is not trivial. A geometrical method, consisting in the reduction of the polygons into right triangles whose analytical solutions of the integrals of the field and the potential can be calculated, has been proposed. Therefore, it is now possible to calculate analytically the scalar potential and all the components of the magnetic field created by any polyhedron-shaped magnet. The results are very useful for the calculation and design of magnetic field sources, sensors and actuators.

\section{REFERENCES}

[1] G. Akoun and J.-P. Yonnet, "3D analytical calculation of the forces exerted between two cuboidal magnets," IEEE Trans. Magn., vol. MAG-20, no. 5, pp. 1962-1964, Sep. 1984.

[2] P. Elies and G. Lemarquand, "Analytical optimization of the torque of a permanent-magnet coaxial synchronous coupling," IEEE Trans. Magn., vol. 34, no. 4, pp. 2267-22731, Jul. 1998.

[3] J.-P. Yonnet, "Permanent magnet bearings and couplings," IEEE Trans. Magn., vol. MAG-17, no. 1, pp. 1169-1173, Jan. 1981

[4] R. Ravaud, G. Lemarquand, S. Babic, V. Lemarquand, and C. Akyel, "Cylindrical magnets and coils: Fields, forces, and inductances," IEEE Trans. Magn., vol. 46, no. 9, pp. 3585-3590, Sep. 2010.

[5] R. Ravaud, G. Lemarquand, V. Lemarquand, and C. Depollier, "Analytical calculation of the magnetic field created by permanent-magnet rings," IEEE Trans. Magn., vol. 44, no. 8, pp. 1982-1989, Aug. 2008.

[6] S. I. Babic and C. Akyel, "Improvement in the analytical calculation of the magnetic field produced by permanent magnet rings," Progress in Electromagn. Res. C, vol. 5, pp. 71-82, 2008.

[7] E. P. Furlani, "Formulas for the force and torque of axial couplings," IEEE Trans. Magn., vol. 29, no. 5, pp. 2295-2301, Sept. 1993.

[8] H. Rakotoarison, J.-P. Yonnet, and B. Delinchant, "Using coulombian approach for modelling scalar potential and magnetic field of a permanent magnet with radial polarization," IEEE Trans. Magn., vol. 43, no. 4, pp. 1261-1264, Apr. 2007.

[9] J. L. J. Janssen, J. J. H. Paulides, and E. A. Lomonova, “3D analytical field calculation using triangular magnet segments applied to a skewed linear permanent magnet actuator," COMPEL, vol. 29, no. 4, pp. 984-993, 2010.

[10] C. Rubeck, J.-P. Yonnet, B. Delinchant, and O. Chadebec, "Calcul du potentiel et du champ magnétostatique créés par un aimant permanent de forme polygonale uniformément aimanté," in Proc. Actes de la Conference Electrotechnique du Futur (EF) 2011, Belfort, France, Dec. 2011 [Online]. Available: (http://hal.archives-ouvertes.fr/hal-00653205)

[11] E. Durand, "Distributions superficielles," in Electrostatique, Tome I-Les Distributions. Paris: Masson et Cie, 1966, pp. 13-225.

[12] D. Wilton, S. Rao, A. Glisson, D. Schaubert, O. Al-Bundak, and C. Butler, "Potential integrals for uniform and linear source distributions on polygonal and polyhedral domains," IEEE Trans. Antennas Propag., vol. 32, no. 3, pp. 276-281, Mar. 1984.

[13] B. Hachi-Ashtiani, "Ecole Centrale Lyon," Thèse de Doctorat, Lyon, France, 1998.

[14] R. Graglia, "On the numerical integration of the linear shape functions times the 3-D Green's function or its gradient on a plane triangle," IEEE Trans. Antennas Propag., vol. 41, no. 10, pp. 1448-1455, Oct. 1993. 